\title{
Comment on Piątkowski et al.: Comparison of different locking plate fixation methods in distal tibia fractures
}

\author{
You Zhou ${ }^{1} \cdot \operatorname{Tao}^{X_{i a o}}{ }^{1}$
}

Received: 7 November 2015 / Accepted: 8 December 2015 / Published online: 19 December 2015

(C) SICOT aisbl 2015

To the Editor,

We read with interest the article by Piątkowski et al. [1] entitled "Comparison of different locking plate fixation methods in distal tibia fractures" published online in July 2015 in International Orthopaedics. In this paper, the authors concluded that outcome of plate fixation of distal tibia fracture did not depend on the fixation method (bridge plating or rigid fixation) or surgical approach (medial or anterior). It is an interesting study. Nevertheless, we have several queries which we would like to communicate to the authors.

Firstly, bias may exist since the investigators did not report if it is the same surgeon who operated on both groups or a different surgeon for each group. Generally, because of different clinical experience, different surgeons can affect the results greatly. We suggest that the authors should record how many surgeons operated on these patients.

Secondly, the authors agreed that skin and soft tissue healing problems were common complications. However, they reported many significant complications like reoperation rate, bone union complications, infection and angular deformity, but did not includ skin and wound healing problems. Consequently, it would be better to assess skin and soft tissue complications.

Thirdly, the investigators did not described whether there are open fracture cases among selected patients. Compared to closed fracture, most surgeon treat open fracture patients in two or three stages [2]. Therefore, management of open frac-

Tao Xiao

xiaotaoxyl@163.com

1 Department of Orthopaedics, The Second Xiangya Hospital of Central South University, Changsha 410011, China tures may have great influence on the outcome. We recommend that they may make it clear in this paper whether open fracture patients are included or not.

Furthermore, when calculating mean time to bone union, the authors chose to include those patients that required reoperation due to non-union. We feel confused about how to calculate if those non-union patients are included. Besides, non-union patients are different from the other patients, which can be a confounding factor to assess time to bone union.

Finally, there was a slip of the pen in the conclusion section. When they analyzed results of problems with bone union between two surgical approach groups, with careful reading, we think the $P$ value should be 0.08 instead of 0.053 .

In conclusion, it is unable to judge easily which surgical approach and method of plate fixation is related to better functional result and lower complication rate. More research is needed in the future. We believe that our remarks will contribute to more accurate elaboration of the results presented by Piątkowski et al.

\section{Compliance with ethical standards}

Conflict of interest The authors declare that they have no conflict of interest.

\section{References}

1. Piatkowski K, Piekarczyk P, Kwiatkowski K, Przybycien M, Chwedczuk B (2015) Comparison of different locking plate fixation methods in distal tibia fractures. Int Orthop 39(11):2245-2251. doi: 10.1007/s00264-015-2906-4

2. Jacob N, Amin A, Giotakis N, Narayan B, Nayagam S, Trompeter AJ (2015) Management of high-energy tibial pilon fractures. Strat traum limb Recon (online). doi:10.1007/s11751-015-0231-5 